\title{
A PREDICTIVE CONTROL APPROACH FOR BIDIRECTIONAL DC-DC POWER CONVERTER IN SUPERCAPACITOR ENERGY STORAGE SYSTEMS
}

\author{
PHAM TUAN ANH ${ }^{1,2}$, NGUYEN VAN CHUONG ${ }^{1}$, CAO XUAN DUC ${ }^{1}$, \\ AND NGUYEN PHUNG QUANG ${ }^{1}$ \\ ${ }^{1}$ Hanoi University of Science and Technology \\ ${ }^{2}$ Vietnam Maritime University; phamtuananh@vimaru.edu.vn
}

\begin{abstract}
A possible solution to mitigate the wind power fluctuations is integrated energy storage systems (ESS) to the wind energy conversion systems (WECS). The supercapacitor ESS (SCESS) is able to smooth out the output power of wind turbine by exchanging bidirectional power between wind turbine and supercapacitor through power conversion system. The SCESS consists of supercapacitor, serving as a DC power source, and power conversion system comprising a bidirectional DC-DC converter and a bidirectional DC-AC converter. Although control methods for a DC-AC converter are almost fully developed, there is not any scientific research for nonlinear control design of DC-DC converter due to the shortage of its nonlinear model describing power exchange process. This paper focuses on a SCESS in terms of modeling and control designing aim to manage active power flow between the grid and the SCESS. A predictive control algorithm for discrete-time bilinear state-space model of a non-isolated bidirectional DC-DC converter is proposed. This algorithm is supplementary methods for this converter besides linear or hysteresis control methods in other research. Simulations validate the effectiveness of the proposed control.
\end{abstract}

Keywords. Supercapacitor energy storage system (SCESS), bidirectional DC-DC converter, voltage source inverter, active rectifier, predictive control.

\section{INTRODUCTION}

Wind power is one of the renewable energy resources that help to lower the global warming trend. Unlike a traditional centralized generation plant, these new sources may be located anywhere on the grid. Islands and densely populated areas finding it hard to be interconnected with the national electric distribution grids rely primarily on Diesel generators as main electric power supply systems for them [1].

The model system used in this study consists of a wind turbine generator (WTG), a diesel generator (Genset), a SCESS and a load forming a wind - diesel - SCESS hybrid power system (WDS - HPS) as shown in Fig 1. Assuming that the power system operates separately and it is isolated from the national electric distribution grids. The WTG output power $P_{\text {wind }}$, SCESS output power $P_{S C E S S}$, the combined power $P_{\text {wind-SCESS }}$ and the diesel generator power $P_{\text {diesel }}$ flow to the system load. Because of wind fluctuation, the connection of a wind turbine to a grid (especially in islands where the grids are weak) could create severe problems to the transmission line designed for constant power and to power system stability. A possible solution to the problem is the use of suitable energy storage system (ESS) $[2,3]$. This approach employs a power conversion system, 


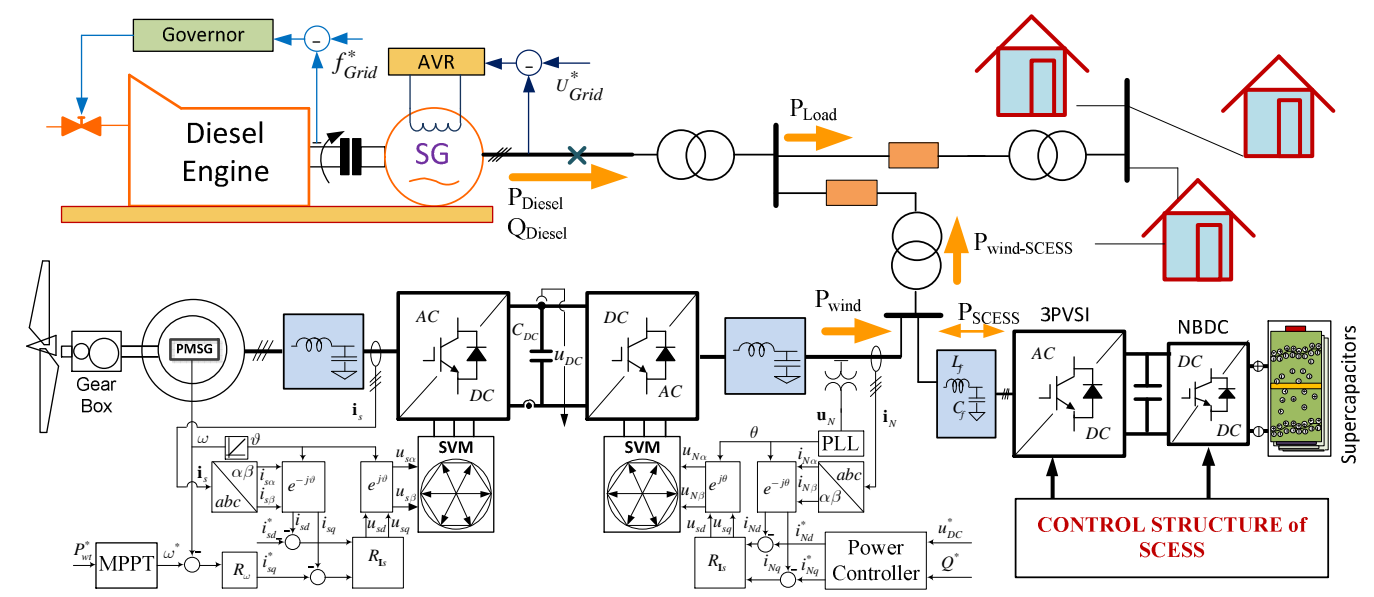

Figure 1: A single-phase diagram of an isolated wind-diesel-energy storage hybrid power system.

which has ability to provide the positive response of fast-dynamic energy storage for high power requirement in order to smooth out the output to eliminate rapid power oscillations on the grid. The main issues of using the ESS to smooth out output power include ESS topology and capacity requirement, converter control, ESS power reference setting, etc. Different types of ESS such as batteries, supercapacitors, flywheels, superconducting magnetic energy storage, etc. are also studied in $[2,4,5]$. Supercapacitors represent one of the innovations in the field of electrical energy storage, which is fulfilling the gap between capacitor and battery. The SC has large capacitance, excellent instantaneous charge-discharge performance, higher power density (but lower energy density) and longer life cycle than a battery.

The control of SCESS has been studied by using conventional linear control techniques (see e.g. [6-9]). However NBDC exhibits a nonlinear behaviour (as presented in next section). The linear control only ensures stability for a certain operation point. This paper proposes a new effective control scheme of SCESS in which a nonlinear MPC control strategy for NBDC cooperates with the conventional linear PI and Dead-beat controller for 3PVSI in order to achieve rapid response of both active and reactive power exchanges.

The paper is organized as follows: Models of the SCESS is introduced in Section 2. In Section 3, an MPC controller for NBDC whose responses are for keeping the constant DC link voltage is designed. The controllers of 3PVSI in charge of active power and unity power factor control designed using familiar instantaneous power theory can be found in $[10,11]$. The effectiveness of the proposed control scheme validated by simulations is shown in Section 4 .

\section{SYSTEM CONFIGURATION AND MODELING}

In this study, the SCESS consists of a three-phase PWM voltage source inverter (3PVSI) connected between the grid interconnection point and the supercapacitor connected to DC link through a nonisolated bidirectional DC-DC converter (NBDC) as shown in Figure 2. The operating principles and modeling of 3PVSI can be found in $[10,11]$. The following presentation focuses on the NBDC only.

The NBDC composes of two IGBTs having anti-parallel diode making up a bi-positional switch. 
In such a bidirectional switching power-pole, the positive inductor current represents a charge mode. Similarly, the negative inductor current represents a discharge mode. In this approach, instead of independently Pulse Width Modulation (PWM) control the power switches (this technique can be found in several studies such as $[12,13]$ ), they are controlled complementarily - by means of $q, \bar{q}$ (the switching signals of $\left.S_{B K}, S_{B S}\right)$.

$$
\left\{\begin{array}{l}
\frac{d \bar{i}_{L}}{d t}=-\frac{R_{L}}{L} \bar{i}_{L}+\frac{1}{L} \bar{u}_{d c} u-\frac{\bar{u}_{s c}}{L} \\
\frac{d \bar{u}_{d c}}{d t}=-\frac{1}{C_{d c}} \bar{i}_{L} u+\frac{\bar{i}_{i n v}}{C_{d c}}
\end{array}\right.
$$

Within any period $T$, there are two sub-intervals: $T_{O N}\left(S_{B K}\right.$ on, $S_{B S}$ off $) ; T_{O F F}\left(S_{B K}\right.$ off $f, S_{B S}$ on $)$ (see [11] for more details). The averaged model of NBDC as (1), where $u(t) \triangleq \frac{T_{O N}(t)}{T}$ is called duty-cycle. The model (1) is bilinear because of product $\bar{u}_{d c} u$ and $\bar{i}_{L} u$. In order to apply MPC (Model Predictive Control) algorithm for NBDC controller, the continuous-time model (1) must be normalized and then converted to discrete-time model.

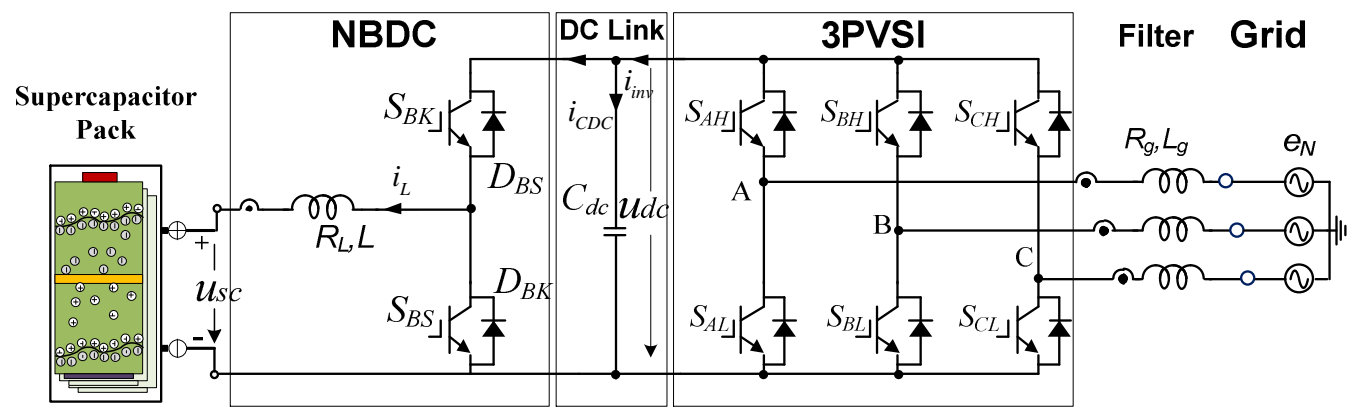

Figure 2: The topology of the SCESS.

\subsection{Normalization}

By setting:

$$
x_{1}=\frac{\bar{i}_{L}}{U_{\text {ref }} \sqrt{\frac{C_{d c}}{L}}} ; x_{2}=\frac{\bar{u}_{d c}}{U_{r e f}} ; x_{3}=\frac{\bar{u}_{s c}}{U_{r e f}} ; i_{c h}=\frac{\bar{i}_{i n v}}{U_{r e f}} \sqrt{\frac{L}{C_{d c}}} ; a=R_{L} \sqrt{\frac{C_{d c}}{L}}
$$

Then the averaged dynamic model of the NBDC after normalizing is presented:

$$
\left\{\begin{array}{l}
\dot{x}_{1}=-a x_{1}+x_{2} u-x_{3} \\
\dot{x}_{2}=-x_{1} u+i_{c h}
\end{array} .\right.
$$

\section{Discrete-time state space model:}

By setting $\mathbf{x}=\left(\begin{array}{l}x_{1} \\ x_{2}\end{array}\right)$ and matrices: $\mathbf{A}_{C}=\left(\begin{array}{cc}-a & 0 \\ 0 & 0\end{array}\right) ; \mathbf{F}_{C}=\left(\begin{array}{cc}0 & 1 \\ -1 & 0\end{array}\right) ; \eta_{C}=\left(\begin{array}{c}-x_{3} \\ i_{c h}\end{array}\right)$ then the equation (2) is re-written as:

$$
\dot{\mathbf{x}}=\left(\mathbf{A}_{C}+\mathbf{F}_{C} u\right) \mathbf{x}+\eta_{C}
$$


In a sample period of time $T$-execution time of microcontroller, the $i_{c h}$ and $x_{3}$ (corresponding to $i_{\text {inv }}, u_{S C}$ ) change very slowly, so $\eta_{C}$ can be considered as a constant value. Let it be integral $\mathbf{x}(t)$ from $k T$ to $(k+1) T$, so:

$$
\mathbf{x}(k+1)=\mathbf{\Phi}(k) \mathbf{x}(k)+\eta_{C}(k) T
$$

In which, the system matrix $\mathbf{\Phi}(k)=e^{\left[\mathbf{A}_{C}+\mathbf{F}_{C} u(k)\right] T}$ is approximated by the first degree Taylor series. Setting $\mathbf{A}=\mathbf{I}+\mathbf{A}_{c} T, \mathbf{F}=\mathbf{F}_{c} T$. and $\eta=\eta_{C} T$, the Eq. (4) becomes:

$$
\mathbf{x}(k+1)=\mathbf{A} \mathbf{x}(k)+\mathbf{F} \mathbf{x}(k) u(k)+\eta(k)
$$

Obviously, it is the discrete - time bilinear state-space model, and the MPC algorithm for the bilinear model will be presented in section 3 .

\section{CONTROL SCHEME OF THE SCESS}

These converters must be controlled in order to meet the following requirements: tight DC bus voltage regulation; can interact with the three-phase AC grid at the point of common coupling; perfect track of active and reactive power of its references. In this approach, there are two control structures of NBDC and 3PVSI that are shown in Figure 3. The current can be either drawn from or injected into the DC-link by the operation mode. It is required to ensure the power balance between the primary power source - supercapacitor and the load by regulating the DC link voltage to a fixed value. The DC-link voltage can be subjected to transient conditions due to the change of the power exchanged by the SCESS. The DC-link voltage control is achieved through the control of NBDC while the controllers of 3PVSI are responsible for tracking the active and reactive power reference values. Internal current and voltage loops in both converters are used.

In fact, the reference value of active power is the high frequency fluctuating components of the demand-generation power mismatch which is given by outer loop control so called "Energy management Algorithm - EMA". The researchers aim to examine the performances of the SCESS; EMA will not be discussed in this paper. Instead, step signal will be used to generate the reference active power of the SCESS.
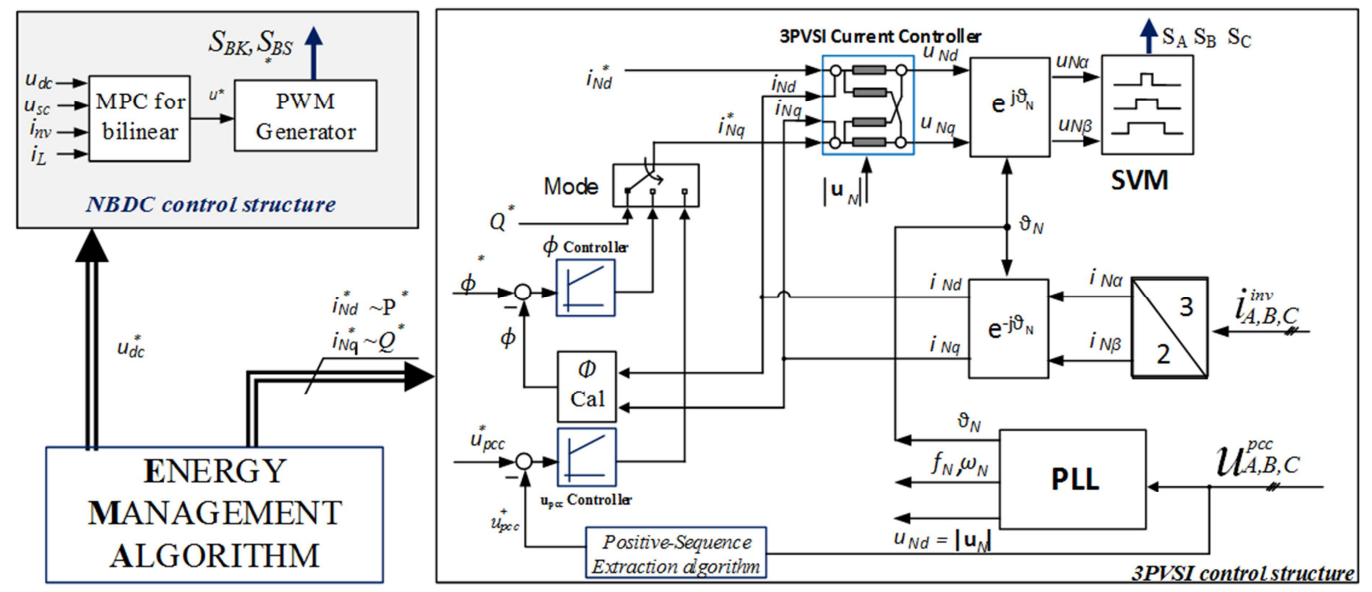

Figure 3: The control structure of the SCESS. 


\subsection{Controller design for NBDC}

A proposed MPC for NBDC can keep $u_{d c}$ as a command value $U_{r e f}^{*}\left(x_{2}^{*}=1\right)$. The MPC structure comprises three parts: predictive model, cost function, and optimization algorithm. The discretetime bilinear state model (5) of NBDC is utilized directly to build the 2 steps predictive model as follows:

$$
\begin{aligned}
\tilde{\mathbf{x}}= & \left(\begin{array}{c}
\mathbf{x}(k+1) \\
\mathbf{x}(k+2)
\end{array}\right) \\
= & \left(\begin{array}{c}
\mathbf{A} \\
\mathbf{A}^{\mathbf{2}}
\end{array}\right) \mathbf{x}(k)+\left(\begin{array}{cc}
\mathbf{F x}(k) & 0 \\
\mathbf{A} \mathbf{F} \mathbf{x}(k) & \mathbf{F A x}(k)+\mathbf{F} \eta(k)
\end{array}\right)\left(\begin{array}{c}
u(k) \\
u(k+1)
\end{array}\right) \\
& +\mathbf{F}^{2} \mathbf{x}(k) u(k) u(k+1)+\left(\begin{array}{c}
\eta(k) \\
\mathbf{A} \eta(k)+\eta(k)
\end{array}\right)
\end{aligned}
$$

The cost function is determined from [14]. In which, the weight factor equal 1

$$
J(k)=\sum_{j=1}^{2}\left\|\mathbf{x}(k+j)-\mathbf{x}_{s}(k+j)\right\|_{Q}^{2}+\sum_{j=1}^{2}\left\|u^{*}(k+j-1)-u_{s}(k+j-1)\right\|_{R}^{2}
$$

In which $\|\mathbf{b}\|_{\psi}^{2}=\mathbf{b}^{T} \psi \mathbf{b} ; \mathbf{x}_{s}, u_{s}$ are steady states at each period of time. Begin with (5), given a set point $y_{s}=x_{2 s}=1$ for the output, the corresponding steady state values for $\left(\mathbf{x}_{s}, u_{s}\right)$ can be found by solving the following equations, in which

$$
a=R_{L} \sqrt{\frac{C_{d c}}{L}}:\left\{\begin{array}{l}
\mathbf{x}(s)=\mathbf{A} \mathbf{x}(s)+\mathbf{F} \mathbf{x}(s) u(s)+\eta(k) \\
y_{s}=x_{2 s}=1
\end{array}\right.
$$

Because duty cycle $u_{s}>0$, the solution $u_{s}(k)=\left(x_{3}(k)-\sqrt{x_{3}^{2}(k)+4 a i_{c h}(k)}\right) / 2<0$ is eliminated,

$$
\Rightarrow\left\{\begin{array}{c}
u_{s}(k)=\left(x_{3}(k)+\sqrt{x_{3}^{2}(k)+4 a i_{c h}(k)}\right) / 2 \\
x_{1 s}(k)=i_{c h}(k) / u_{s}(k)
\end{array}\right.
$$

then the optimization algorithm used here is "optimization algorithm based on fixed search directions" [14]. The predictive future states are represented separately to 2 directions $u(k)$ and $u(k+1)$ :

$$
\tilde{\mathbf{x}}=\left(\begin{array}{c}
\mathbf{x}(k+1) \\
\mathbf{x}(k+2)
\end{array}\right)=\mathbf{K}_{1}+\mathbf{V}_{1} u(k)=\mathbf{K}_{2}+\mathbf{V}_{2} u(k+1),
$$

where, matrices $\mathbf{K}_{i}, \mathbf{V}_{i}(i=\overline{1,2})$ are given by:

$$
\begin{aligned}
& \mathbf{K}_{i}=\tilde{\mathbf{x}}[u(k+i-1)=0] ; \\
& \mathbf{V}_{i}=\tilde{\mathbf{x}}[u(k+i-1)=1]-\mathbf{K}_{i}
\end{aligned}
$$

And the optimal values for each direction as the following:

$$
\begin{gathered}
u^{*}(k)=-\left(\mathbf{V}_{1}^{T} \tilde{\mathbf{Q}} \mathbf{V}_{1}+\mathbf{R}\right)^{-1}\left(\mathbf{V}_{1}^{T} \tilde{\mathbf{Q}}\left[\mathbf{K}_{1}-\tilde{\mathbf{x}}_{s}\right]-\mathbf{R} u_{s}(k)\right), \\
u^{*}(k+1)=-\left(\mathbf{V}_{\mathbf{2}} \tilde{\mathbf{Q}} \mathbf{V}_{\mathbf{2}}+\mathbf{R}\right)^{-1}\left(\mathbf{V}_{2}^{T} \tilde{\mathbf{Q}}\left[\mathbf{K}_{2}-\tilde{\mathbf{x}}_{s}\right]-\mathbf{R} u_{s}(k+1)\right) .
\end{gathered}
$$




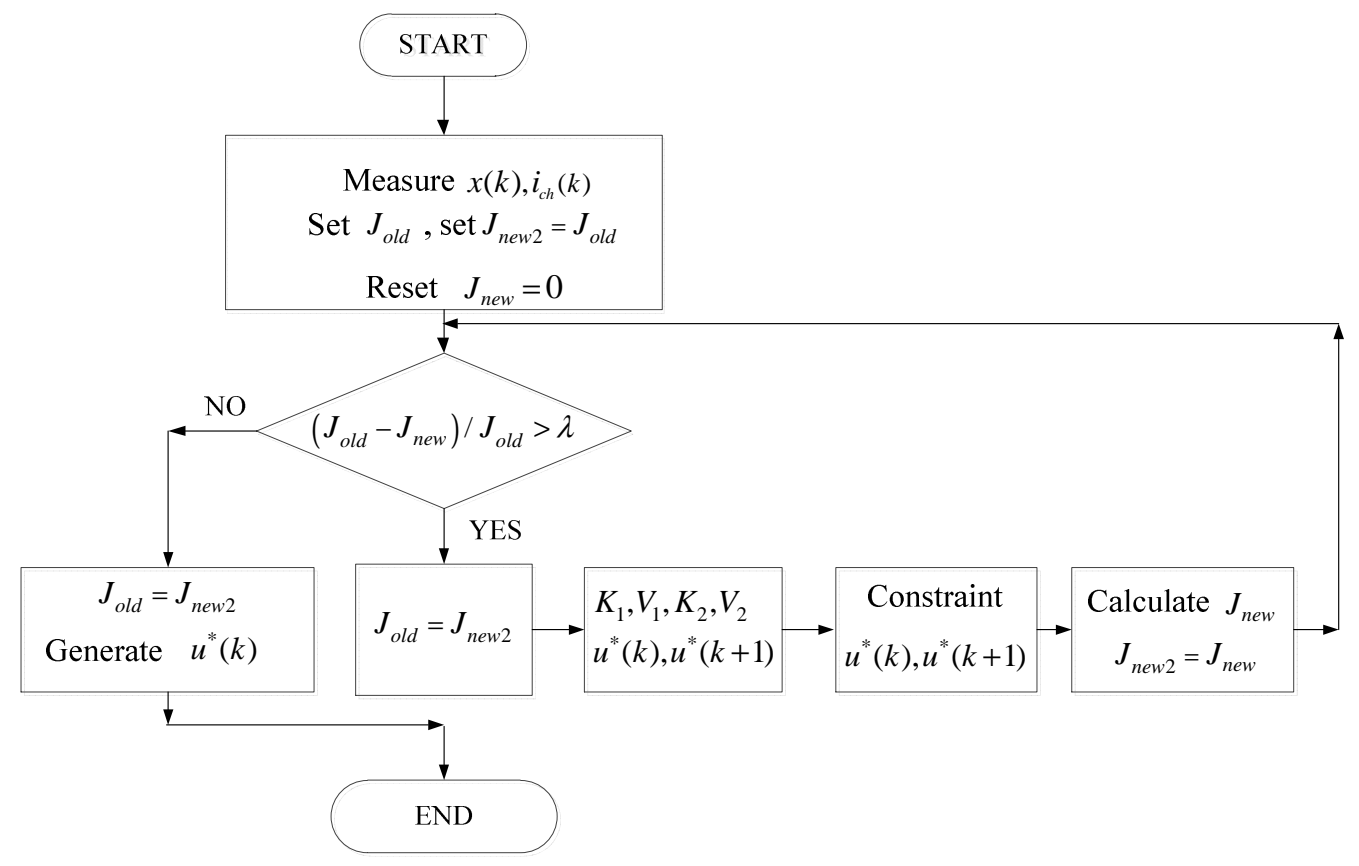

Figure 4: The flow chart of MPC algorithm.

The flow chart of MPC algorithm is shown in Figure 4. The while loop in each the period of time will be stopped if the relative decrease of the cost function is smaller than specified small positive $\lambda$. The smaller value of $\lambda$, the better control quality; but it causes the longer time for execution. In this paper, $\lambda=0.002$. Because not all the inputs have the same impact on the value of cost function $J$, the advantages of this approach of evaluation is that stop criterion is evaluated only after successively optimizing once in every direction $u(k+i-1)$.

\subsection{Controller design for 3PVSI}

Figure 3 shows the control structure of 3PVSI. The power control of the 3PVSI is based on the instantaneous power theory. Voltage Oriented Control (VOC) is based on the use of a $d q$ frame rotating at $\omega$ speed and oriented such that the $d$ axis is aligned on the grid voltage vector. The reference current $d$ component $i_{N d}^{*}$ is controlled to manage active power exchange while the reference current $q$ component $i_{N q}^{*}$ is in charge of reactive power control and typically to obtain a unity power factor. In fact, to have the grid current vector in phase with grid voltage, the vector $i_{N q}^{*}$ should be zero. Assuming that the $d$ axis is perfectly aligned with grid voltage $e_{q}=0$, the active power and reactive power will therefore be proportional to $i_{N d}$ and $i_{N q}$, respectively:

$$
\begin{aligned}
& P=\frac{3}{2} u_{d} i_{N d} \\
& Q=-\frac{3}{2} u_{d} i_{N q}
\end{aligned}
$$

The commands $i_{N d}$ and $i_{N q}$ are realized by an inner-loop current control which plays a decisive role in the control concept and for its correct realization. Therefore, current controllers were designed based on the Bead-beat method as described in $[10,11,15]$ to improve the current rapid response. 


\section{SIMULATION}

\subsection{Simulation Parameters}

A simulation model is built and parameters are: $U_{d c-r e f}=700 \mathrm{~V}, C_{d c}=1000 \mathrm{uF}, C_{c e l l}=58 \mathrm{~F}$, $E S R_{\text {cell }}=0.022 \Omega, N_{\text {series }}=42, N_{\text {parallel }}=8, C_{S C E S S}=10.75 F, E S R_{S C E S S}=0.1155 \Omega$, $\lambda=0.002, U_{\text {cell }}=15 \mathrm{nV}, T=0.0005 \mathrm{~s}$. Valves are ideal. The numerical simulations are carried out with Matlab/Simulink/SimPowerSystems and PLECS. The supercapacitor block in SimPowerSystems is a generic model parameterized to represent most popular types of supercapacitors. The block employs Stern equation and Tafel equation as in [16].

\subsection{Simulation Results}

There are two cases of study simulation: the NBDC independently works and cooperates with 3PVSI in the SCESS.

\subsubsection{The NBDC independently working}

In this bench, the current $i_{i n v}$ which caused by active power exchange via 3PVSI is assumed as Figure 5. There are two sub-intervals: $0 \mathrm{~s}-2 \mathrm{~s}$ demonstrates for charging mode $\left(i_{\text {inv }}>0\right)$ with $u_{d c-r e f}=700 \mathrm{~V}, 2 \mathrm{~s}-4 \mathrm{~s}$ demonstrates for discharging mode $\left(i_{i n v}<0\right)$ with $u_{d c-r e f}=800 \mathrm{~V}$. While the reference DC-link voltage $u_{d c-r e f}$ is changed and the inverter current varies in step form as in Figure 5, the DC-link voltage is perfectly regulated as its desired values (Figure 6). Figure 7 shows the value of cost function $J$ from (7). Its decrease to zero proves the effectiveness of proposed algorithm. Figure 8 illustrates the voltage of supercapacitor $u_{s c}$ in each sub-interval.

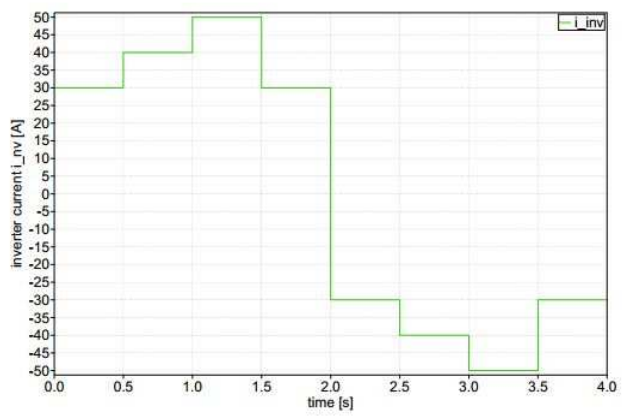

Figure 5: The test signal of current $i_{i n v}$.

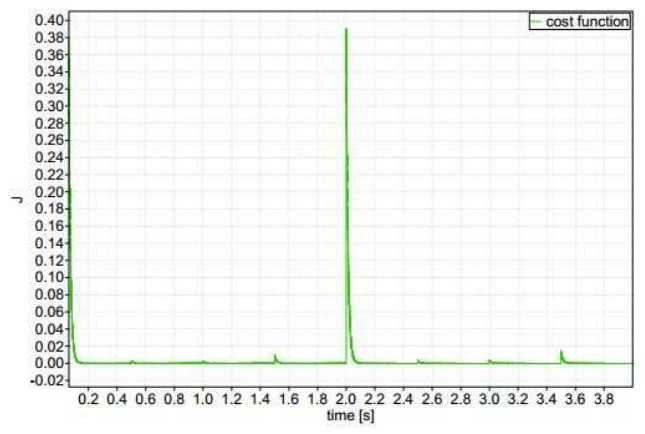

Figure 7: The value of cost function $J$.

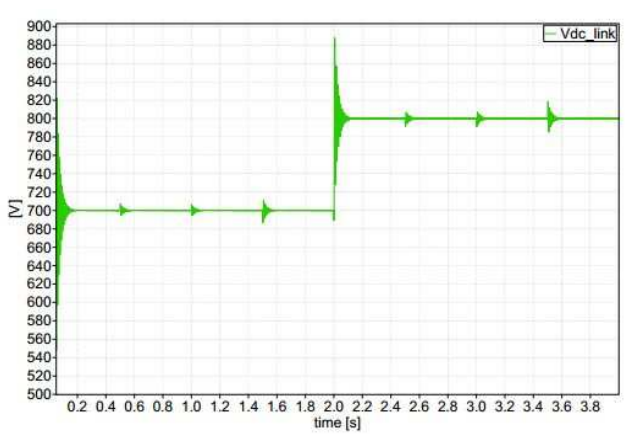

Figure 6: The DC-link voltage response.

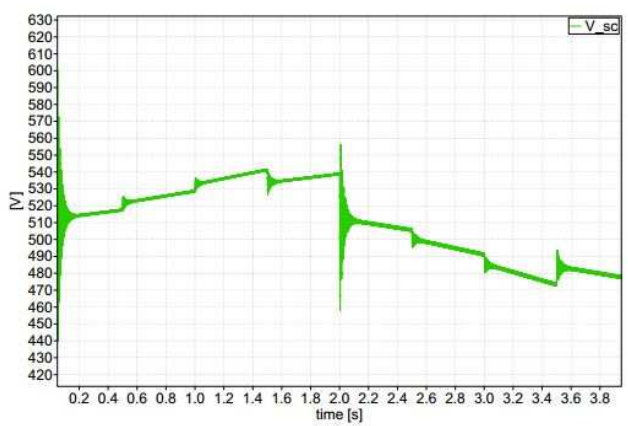

Figure 8: The voltage of supercapacitor. 


\subsubsection{The NBDC cooperates with 3PVSI}

One of the key variables in overall system performance is the active power exchange (proportional to the inductor current of the NBDC and the direct current component of VSI as well) between SCESS and the grid. Thus, its value has to be tightly controlled. Figure 9 shows the inverter current $i_{\text {inv }}$ response to a step-change active power exchange illustrating the charge mode (Positive inductor current value) and discharge mode (Negative inductor current value). Figure 10 and Figure 11 illustrate that: while active power is exchanged (SCESS alternated between charge mode and discharge mode), the voltage of the DC link is kept constant. Figure 12 explains the dynamic performances of the supercapacitor during charging and discharging process in terms of energy (stateof-charge).

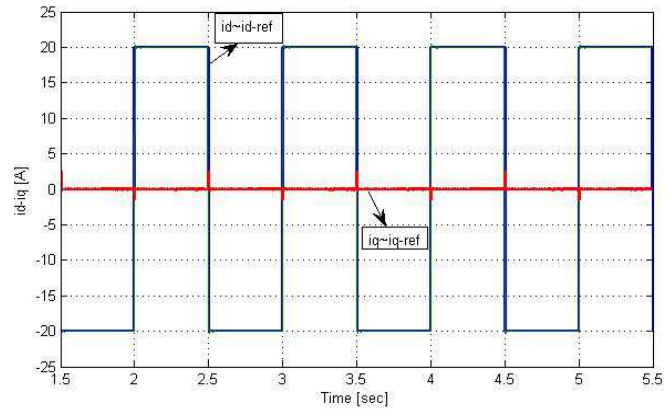

Figure 9: $d q$ components of current response of 3PVSI.

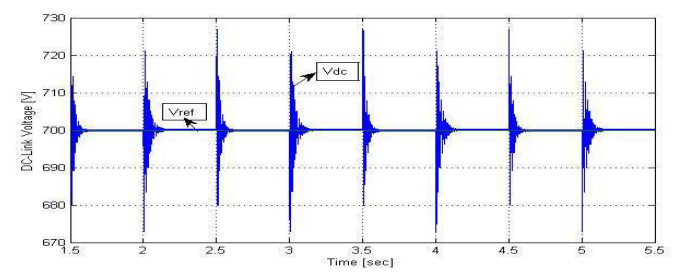

Figure 11: DC link voltage response.

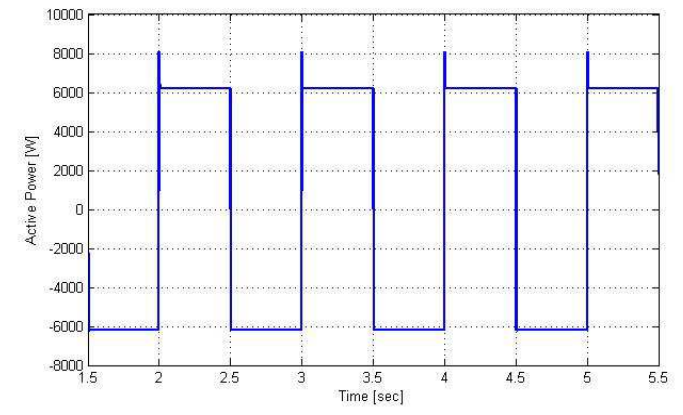

Figure 10: Active Power exchange response of the SCESS.

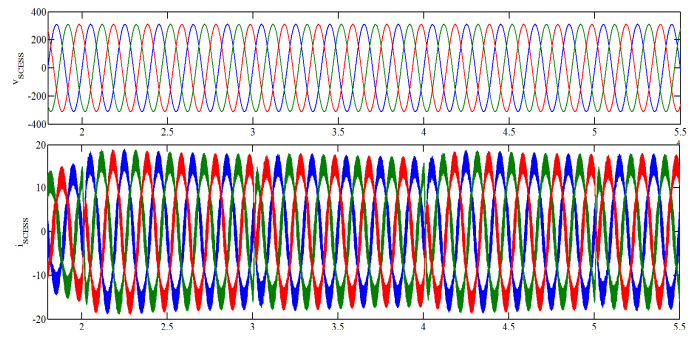

Figure 12: Three phase voltage-current of SCESS.

In order to show the effectiveness of control structure of SCESS with proposed predictive control approach in smoothing out the output power of wind turbine, the wind-diesel-SCESS hybrid power system is established. Figure 13 shows the shape of total power $P_{\text {wind-SCESS }}$ which is much more stable than the shape of wind power.

In the literature, some control approaches are applied to NBDC for specific applications. However, these approaches have some drawbacks. For example, a new PI controller design for both two modes of NBDC in Electrical Vehicle application [17], but it causes high fluctuation around a set point and long settling time. Another example is sliding mode approach for NBDC in supercapacitor automotive application [18], in which it's required buck and boost mode are controlled separately by two controllers. The proposed MPC algorithm in this paper solves these problems. Figure 14 shows 


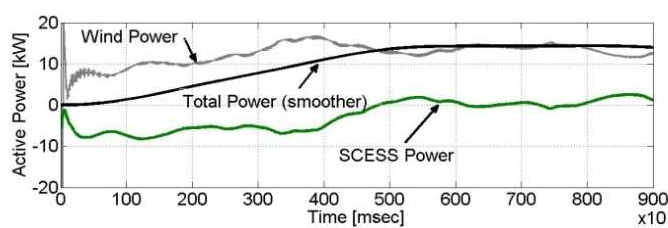

Figure 13: Smoothing out active power.

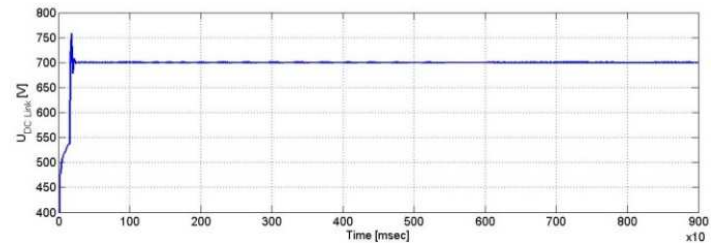

Figure 14: DC-link voltage.

that this algorithm brings lower overvoltage around a set point, fasten response time, and especially, only one controller is used to control both two modes.

\section{CONCLUSION}

The DC-link voltage can be subjected to transient conditions due to the change of power exchanged by the SCESS. The objective control of DC-link voltage which ensures the balancing of active power exchange between the SCESS and the grid is archived. The perfectly tracking performance lets us confirm that the proposed nonlinear MPC algorithm of non-isolated bidirectional DC-DC converter creates good condition to integrate the SCESS with a wind power system to mitigate the power fluctuation.

\section{REFERENCES}

[1] J. K. Kaldellis, "Stand-alone and hybrid wind energy systems," ed: Woodhead Publishing Limited, 2010.

[2] F. Díaz-González, A. Sumper, O. Gomis-Bellmunt, and R. Villafáfila-Robles, "A review of energy storage technologies for wind power applications," Renewable and Sustainable Energy Reviews, vol. 16, pp. 2154-2171, 2012.

[3] P. T. Anh and N. P. Quang, "Energy storage system for wind power plants - system structure, operating mode and technical problems," in CD Proc. of the 6th Vietnam Conf. on Mechatronics VCM-2012, Hanoi, 2012, pp. 107-114.

[4] S. B. g. Energie, "Energy storage technologies for wind power integration", Université Libre de Bruxelles, 2010.

[5] D. Connolly, "A review of energy storage technologies: for the integration of fluctuating renewable energy," Tech. Rep., ed: University of Limerick, 2010.

[6] S. I. Gkavanoudis and C. S. Demoulias, "A combined fault ride-through and power smoothing control method for full-converter wind turbines employing Supercapacitor Energy Storage System," Electric Power Systems Research, vol. 106, pp. 62- 72, 2014.

[7] N. Mendis, K. Muttaqi, and S. Perera, "Active power management of a super capacitor-battery hybrid energy storage system for standalone operation of DFIG based wind turbines," in Industry Applications Society Annual Meeting (IAS), 2012 IEEE. IEEE, 2012, pp. 1-8.

[8] N. Mendis and K. Muttaqi, "An integrated control approach for standalone operation of a hybridised wind turbine generating system with maximum power extraction capability," Electrical Power and Energy Systems, vol. 49, pp. 339-348, 2013. 
[9] L. Qu and W. Qiao, "Constant power control and fault-ride-through enhancement of DFIG wind turbines with energy storage," in Industry Applications Society Annual Meeting, 2009. IAS 2009. IEEE. IEEE, 2009.

[10] R. Teodorescu, M. Liserre, and P. Rodriguez, Grid converters for photovoltaic and wind power systems. John Wiley \& Sons, 2011, vol. 29.

[11] N. P. Quang, Lecturer notes on Control of Power Electronics: Hanoi University of Science and Technology, 2012.

[12] M. G. Molina, Dynamic modelling and control design of advanced energy storage for power system applications. INTECH Open Access Publisher, 2010. Url: http://www.intechopen.com/books/dynamic-modelling/dynamic-modelling-and-controldesign-of-advanced-energy-storage-for-power-system-applications

[13] S. C. W. Kramer, B. Kroposki, and H. Thomas, "Advanced Power Electronic Interfaces for Distributed Energy Systems," Technical Report NREL/TP-581-42672 March 2008, 2008.

[14] H. Bloemen, T. van den Boom, and H. Verbruggen, "Optimization algorithms for bilinear model - based predictive control problems," AIChE journal, vol. 50, no. 7, pp. 1453-1461, 2004.

[15] N. P. Quang and J.-A. Dittrich, "Vector control of three phase AC machine- System Development in the Practice," Springer, Berlin - Heidelberg, 2008.

[16] I. The MathWorks. (2014). Implement generic supercapacitor model.

[17] Zhang Xuhui, Xhui Wen, Zhao Feng, and Guo Xinhua, "A new control strategy for Bidirectional DC-DC converter in Electri Vehicle", International Conference on Electrical Machines and Systems (ICEMS), Beijing, Aug. 2011, pp. 1-4.

[18] H.Maker, H. Gualous, R.Outbib and Monica E.Romero, "Control of bidirectional DC-DC converter for Supercapacitor Automotive Application", downloaded from scribd.com, January. 22, 2008.

Received on August 08, 2014

Revised on June 14, 2015 